\title{
Area Spectroscopy of the Extended Emission Line Region in the Seyfert Galaxy NGC 3516
}

\author{
K. Aoki, H. Ohtani and G. Kosugi ${ }^{1}$ \\ Department of Astronomy, Kyoto University, Kyoto 606-01 JAPAN
}

\section{Yoshida}

Okayama Astrophysical Observatory, National Astronomical

Observatory, Okayama, 719-02 JAPAN

\section{Introduction}

The researches into extended emission line region (EELR) give us the valuable informations about the nucleus. A recent study of the EELR in the Seyfert 1 galaxy NGC 4151 by Yoshida and Ohtani (1993) have revealed that a strongly asymmetric radiation field in this typical Seyfert 1 . They have suggested the anisotropy may be caused inside the BLR. It is important to examine if any other Seyfert 1 galaxy has an anisotropic nuclear radiation.

The famous Seyfert 1 galaxy NGC 3516 has EELR which extends $\sim 10^{\prime \prime}$ on either side of the nucleus. The morphology of the EELR is Z-shape. This nearby $(D=38.9 \mathrm{Mpc})$ galaxy is of type $\mathrm{SB} 0$ for which the object is expected to be free from contamination by HII regions and dust clouds.

\section{Observations and Reduction}

The observations were made with the $1.88 \mathrm{~m}$ telescope at the Okayama Astrophysical Observatory in February 1992, attached with Spectronebulagraph. Details of the instrument are described in the contribution by Ohtani in this proceedings.

An area of $14.4^{\prime \prime}$ width was scanned by the five-arcmin-long slit with P.A. $30^{\circ}$ to cover the Z-shaped component around the nucleus and the southern end of the northern detached component of the EELR. The latter component lies apart by $20^{\prime \prime}(4 \mathrm{kpc})$ from the nucleus.

Another scan of a narrower area was made with P.A. $7^{\circ}$ to cover a nuclear region and the northern detached component.

\section{Results}

From the resulting data cube, we reproduced pure narrow emission line images of $\mathrm{H} \beta,[\mathrm{OIII}], \mathrm{H} \alpha,[\mathrm{NII}]$ and [SII]. From these images, it has been found that the

\footnotetext{
${ }^{1}$ Present address : Department of Astronomical Science, The Graduate University for Advanced Studies, National Astronomical Observatory, Mitaka, Tokyo 181
} 
excitation is higher toward the northern detached component from the nucleus and probably toward the opposite direction.

Using intensity ratios of the emission lines, we examined the ionization mechanism on the diagnosis diagrams to conclude the northern detached component is ionized by a power law ionizing radiation as well as the Z-shaped component.

We also determined the the electron density of the EELR from the line ratio [S II] $\lambda 6716 / \lambda 6731$. A trend of decrease of density with distance is obvious.

\section{Discussion and Conclusion}

The budget of the ionizing photon flux suggests that there is considerable deficit toward the line of site (Fig. 1) in spite that the broad line region is clearly seen from us. Considering the fact that the excitation is higher in the selected direction in the EELR, it seems that collimation of the ionizing radiation from the nucleus is caused in or inside the broad line region. Thus, a simple version of the current unified scheme of Seyfert nuclei may not apply to this typical Seyfert 1 galaxy as in the case of the NGC 4151.

More details are discussed elsewhere (Aoki et al. 1994).

\section{References}

Aoki, K. et al. 1994, submitted to PASJ

Yoshida, M., \& Ohtani, H. 1993, PASJ, 45, 407

\section{EELR}

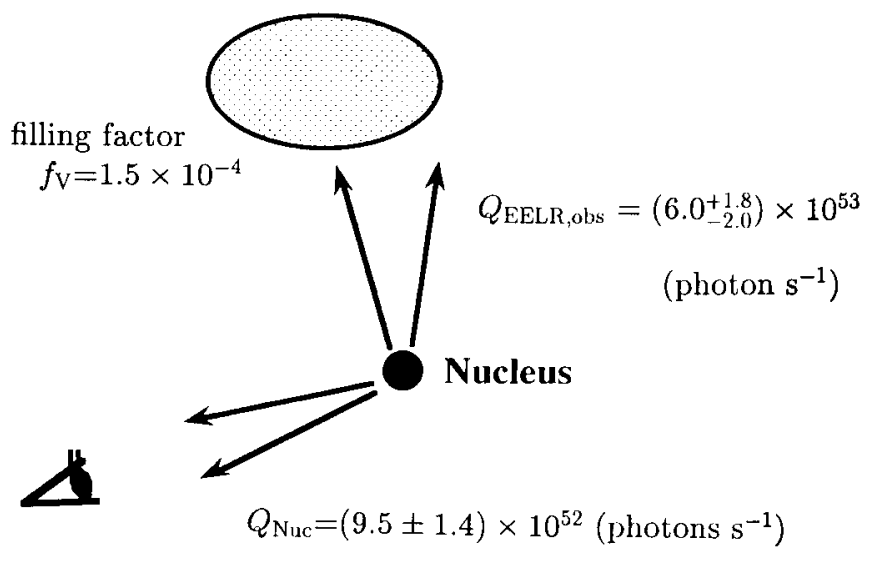

Fig. 1

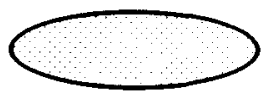

